\title{
Status of the Virgo gravitational-wave detector and the 03 Observing Run
}

\author{
Diego Bersanetti*, on behalf of the Virgo Collaboration \\ INFN Genova \\ E-mail: diego.bersanettiege.infn.it
}

\begin{abstract}
On April 1st 2019 the Virgo gravitational-wave detector, jointly with the two US-based LIGO detectors in Hanford and Livingston, entered the $\mathrm{O} 3$ Observing Run, which is foreseen to last one calendar year. After the past $O 2$ Run, which was characterized by the first ever revelation of gravitational waves emitted by the coalescence of a binary neutron star system, all three detectors underwent an intense, one-year-long Upgrade \& Commissioning phase, aimed at the improvement of the sensitivity and at the increase of the duty cycle. An important difference with respect to the prior Observing Run is the introduction of the Open Public Alert system, which is automatically providing low-latency information for events and superevents to the EM partners, increasing the possibility of a joint detection of events of astrophysical importance in the framework of multimessenger astronomy. This talk will present an overview of the Upgrade \& Commissioning phase of the Virgo detector which preceded the $O 3$ Observing Run, and the detector's current status during the first part of the Run. An overview of the scientific results obtained so far will also also presented. Finally, the current plans and expectations for the remaining part of the Run will be described, followed by a short overview of the future plans for the Virgo detector in the post-O3 phase.
\end{abstract}

European Physical Society Conference on High Energy Physics - EPS-HEP2019 -

10-17 July, 2019

Ghent, Belgium

${ }^{*}$ Speaker. 


\section{Introduction}

The detection of gravitational waves is based on the Michelson interferometer as the working principle, with many technical enhancements which are used in order to increase the sensitivity and reduce the noise. In such an instrument a monochromatic laser beam is splitted, then sent along each arm; the two beams recombine then at the level of the beam splitter before reaching the detection photodiode, which is placed at the anti-symmetric port. When the lengths of the two arms are correctly set in the working point, in absence of gravitational waves the two beams cancel each other out and a null signal is detected; in case of the passage of a gravitational wave, the phase information in the two beams will be different, and the recombination will produce a signal which will then be detected. This information about the phase difference between the light travelling in the two arms is then converted into a length information and, finally, into the gravitational strain $h(t)$.

Advanced Virgo is one of the main gravitational-wave interferometers in the world; together with the two LIGO interferometers in the USA (one in Livingston, LA and one in Hanford, WA) they form a joint network which operates on a global scale. Advanced Virgo (which is located in Cascina, near Pisa, in Italy) is the result of the work of the Virgo Collaboration, which is an European collaboration of eight countries: Italy, France (the two founders), The Netherlands, Poland, Hungary, Spain, Belgium and Germany.

Advanced Virgo joined the international network on August 1st 2017, towards the end of the O2 Observing Run; despite having a lower sensitivity with respect to the LIGO detectors, the Virgo contribution to the network SNR and sky localization was very important. During the last part of the $\mathrm{O} 2$ Observing Run, the interferometer network detected two important gravitational waves: GW170814 [1], a three-detector gravitational signal due to the inspiral and merger of a binary black hole system and, more importantly, GW170817 [2], a three-detector gravitational signal originated by the coalescence, inspiral and merger of a binary neutron star system.

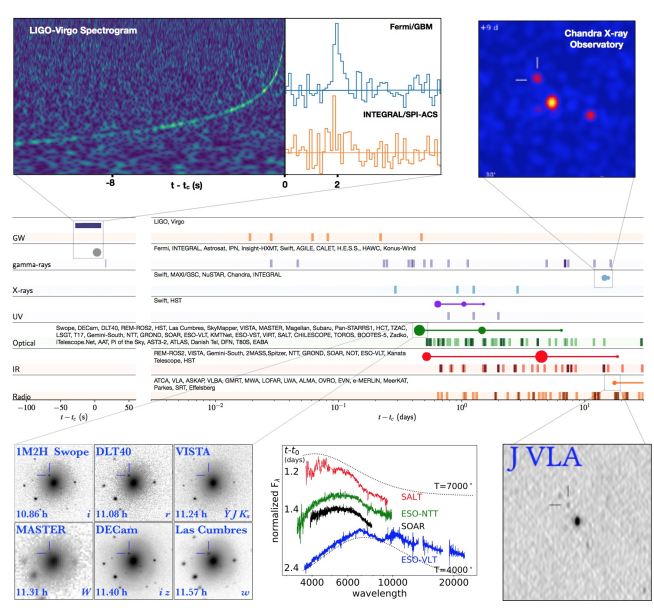

Figure 1: Multi-messenger information about the GW170817 gravitational event, the inspiral and merger of a binary neutron star system.

GW170817 was the first detection ever of gravitational waves produced by the inspiral and merger of a binary neutron star system; given the nature of the source, also EM, optical and gamma- 
ray emissions were detected by several instruments around the world, which were alerted by the gravitational interferometeric network about a possible signal (cfr. Figure 1); in particular, the gamma-ray emission labeled as GRB170817A confirmed the localization of the source as belonging to the galaxy NGC 4993. This event was of a paramount importance, and it marked the birth of the multi-messenger astronomy.

This was only the last of a series of gravitational waves detections which happened during the preceding two years; the full list and data of all such events has been publicly released as the Gravitational Waves Transient Catalog 1 (GWTC-1, [3]); from the characteristics of the several signals it is once more evident that having a network of interferometers is crucial for what concerns sky localization of the sources.

\section{Upgrades \& Commissioning for the $O 3$ Observing Run}

After the end of the $\mathrm{O} 2$ Observing Run the Virgo detector (together with the two LIGO ones) underwent a long Upgrade \& Commissioning phase, which lasted 18 months; this was aimed at the increase of the sensitivity of the three detectors and the preparation for the following $\mathrm{O} 3 \mathrm{Ob}$ serving Run; specifically, the main upgrades done on the Virgo detector were the following: the installation of the monolithic suspensions, the increase of the input laser power and the addition of the squeezing system to the detector. The first upgrade was aimed at the reduction of the thermal noise of the suspensions, while both the input laser power and the squeezing were aimed, albeit in different ways, to the reduction of the quantum noise.

\subsection{Installation of the Monolithic Suspensions}

In the Virgo detector the mirrors are attached to the upper stage of the suspension, the payload, via four thin fibers; the design configuration foresaw the use of fused silica $\left(\mathrm{SiO}_{2}\right)$ fibers, which produce a lesser level of thermal noise with respect to steel wires which are, in contrast, much more robust. During the Commissioning phase preceding the $\mathrm{O} 2$ Run Advanced Virgo suffered several breakages of such fibers (which are used only for the four cavity mirrors), so the configuration was reverted to use steel wires instead.

During the Commissioning phase preceding the $\mathrm{O} 3$ Run the ultimate cause of such breakages was found in the contamination of dust particles coming from the vacuum system; this was mitigated by the cleaning of the vacuum system and the installation of a separated venting pipe for this purpose. The fused silica fibers were then re-installed and no breakages happened since. Therefore, for the $O 3$ Run Advanced Virgo could profit of the better thermal noise level this material provides, which translates in a better sensitivity curve at low frequency.

\subsection{Increase of the Input Laser Power}

One other major upgrade of the Virgo detector was the increase of the laser power entering the interferometer; this was increased from the value used in the $O 2 \mathrm{Run}, 13 \mathrm{~W}$, to a final power of $18 \mathrm{~W}$, with an increase of almost $40 \%$. The net effect of such upgrade is the natural reduction of the shot noise at high frequency, at the cost of the increase of the radiation pressure noise at low frequency and the possible appearance of thermal issues due to the heating of the mirrors; looking, however, at the design sensitivity curve for Advanced Virgo in Figure 2, it can be seen 
that such increase would not make the low frequency noise any worse, as the current projected sensitivity is limited by the thermal noise instead, so the overall net effect is only the beneficial reduction of the shot noise at high frequency. The impact of the thermal effects was limited, and correctly compensated by the Thermal Compensation System, by actuating on both the Input and End mirrors of the arm cavities.

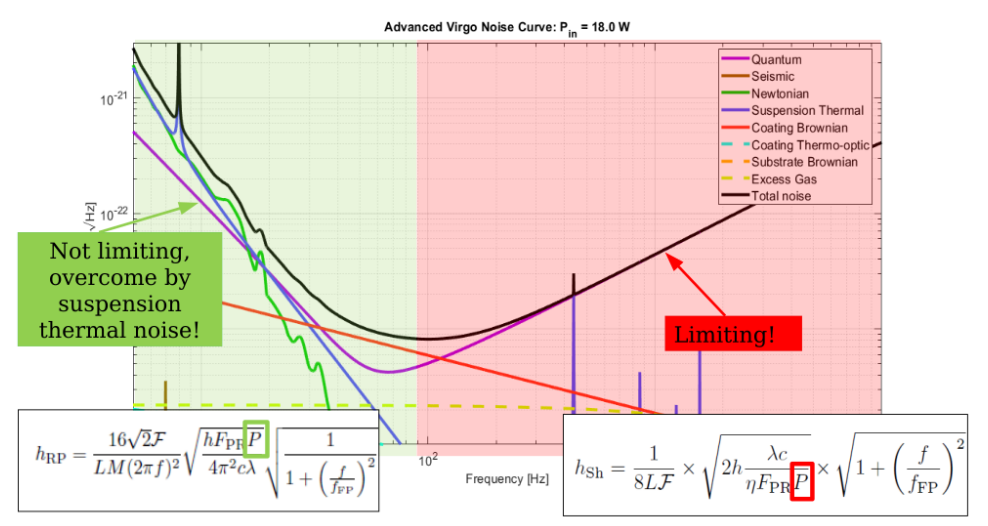

Figure 2: Impact of the quantum noise on the sensitivity curve of Advanced Virgo.

\subsection{Addition of the Squeezing System}

Another way to reduce the shot noise at high frequency is the injection of squeezed vacuum states in the interferometer [4]: following the Heisenberg principle, for a standard ("coherent") state the uncertainty on both quadratures $\hat{X}_{1}$ and $\hat{X}_{2}$ is the same (cfr. Figure 3); using "squeezed" vacuum states, it is possible to reduce the uncertainty on the quadrature of interest (in our case, the phase of the field), at the cost of increasing the other one (the amplitude of the field).

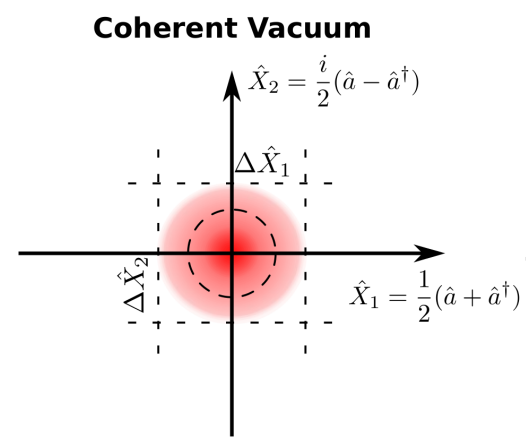

\section{Squeezed Vacuum}

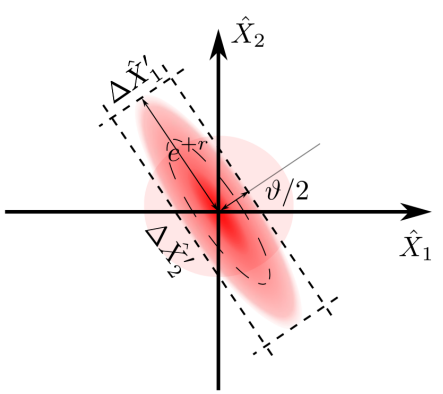

Figure 3: Representation of the uncertainty of coherent and squeezed vacuum states.

The effect on the sensitivity of an interferometer is someway similar to the increase of the input laser power, meaning that the shot noise is reduced, moreover without the appearance of any thermal effect. The cost, related to the increased uncertainty on the amplitude of the field (called anti-squeezing), is the increased noise at low frequency, effect which is masked by the limiting noise in such region, which is the thermal noise of the suspensions. 
The squeezer source which was installed and integrated in the Virgo detector was kindly provided by the Max Planck Institute for Gravitational Physics (AEI-Hannover), which operated it for a long time in the GEO600 gravitational-wave interferometer.

In Figure 4 there is the comparison of the measured sensitivity curve in Advanced Virgo with and without the squeezing system engaged.

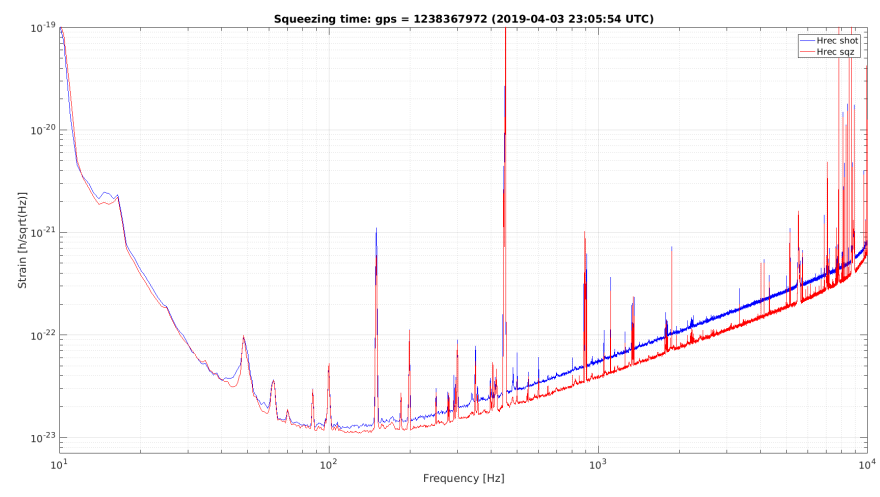

Figure 4: Advanced Virgo sensitivity without (in blue) and with (in red) the squeezing system engaged.

\section{Status of Advanced Virgo in $O 3$}

On April 1st 2019 all the three main gravitational-wave interferometers, Advanced Virgo and the two Advanced LIGOs, went back online and started the $O 3$ Observing Run, which is foreseen to last one calendar year; all the detectors are considered as in the final configuration for data taking, albeit small time frames are periodically still devoted to commissioning, in order to perform small tasks and improvements.

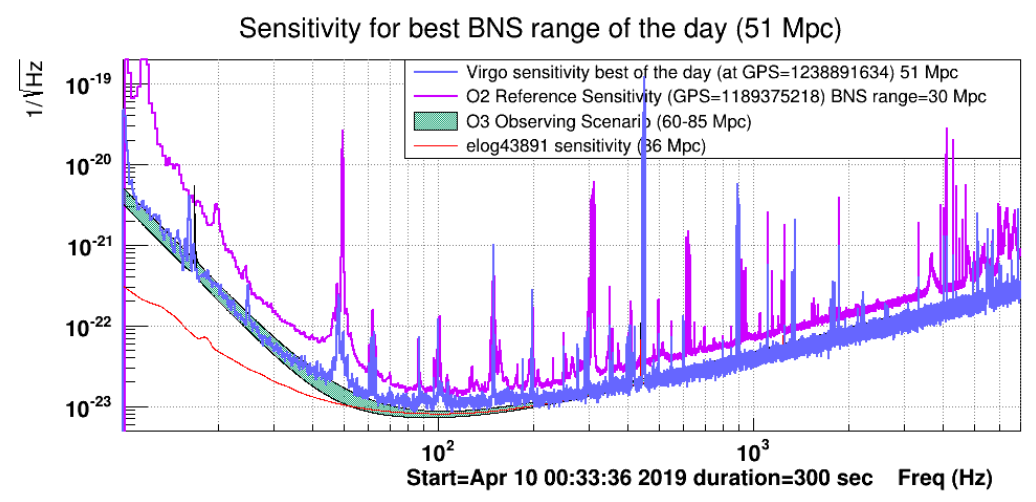

Figure 5: Advanced Virgo sensitivity in the $O 3$ Run.

Advanced Virgo started the Run with a BNS Range ${ }^{1}$ of $\simeq 51 \mathrm{Mpc}$ (Figure 6), quite close to the target for O3, which was set at $60 \mathrm{Mpc}$; Advanced Virgo still suffers from some gap with respect to Advanced LIGO, but this is reduced with respect to the previous Run.

\footnotetext{
${ }^{1}$ The BNS Range is the standard figure of merit for the performance, and it represents the distance at which a gravitational wave from a benchmark binary neutron star system can be detected.
} 
In addition to the increase of the Range, Advanced Virgo performance during $O 3$ proved to be quite good: the BNS Range has been quite stable in the first part of the Run, and at the same time the duty cycle in Science Mode (i.e. with the detector in observing mode) has been high, with an overall $80 \%$, the best value in the whole network (cfr. Figure 6).

As it was stated before, it is maybe more important to evaluate the performances of the interferometric network as a whole: Figure 7 (left panel) shows the network duty cycle with respect to the several configurations of the network: the triple-detector duty cycle is around $45 \%$, which is a very good achievement, which is followed by an overall $36 \%$ when two of the three detectors were observing at the same time; the time fraction when only one, or no detector was online is very low.
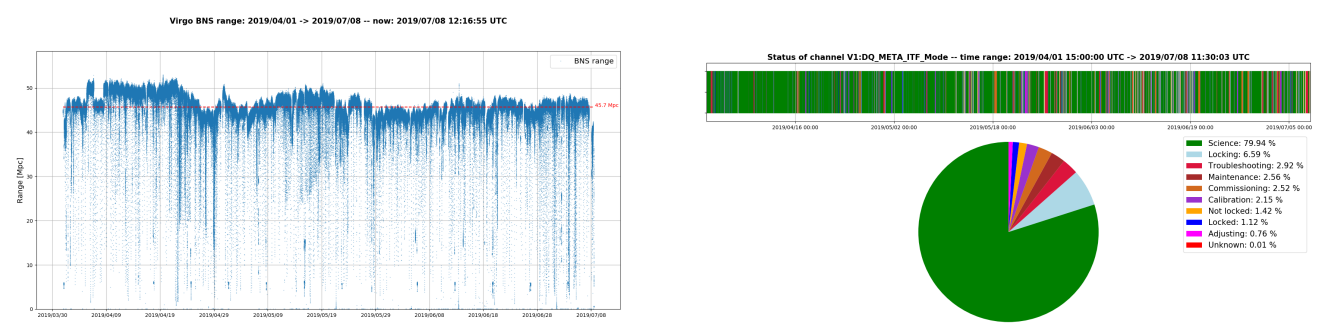

Figure 6: Advanced Virgo BNS Range and duty cycle during the $O 3$ Run.

This was possible also because the three interferometers were kept as synchronized as possible, performing most of the activities not related with Observing Mode (like, for example, the Commissioning activities) at the same time, despite the different time zones of the three detectors (Figure 7, right panel).

Another very important novelty of the $O 3$ Run is the establishment of the Open Public Alert system, by which basic information about candidate Events and Superevents is automatically published and made available to anyone through the Gravitational Wave Candidate Event Database [5]. Each candidate event is selected with automatic triggers and pipelines, and so far about one event per week has been published via this tool.

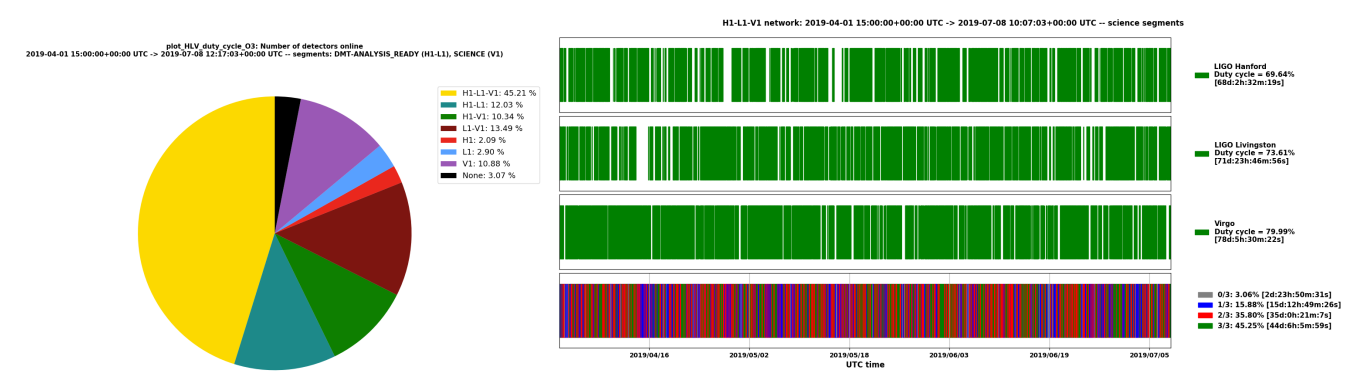

Figure 7: Duty cycle and science segments of the network during the $O 3$ Run.

\section{After O3: Advanced Virgo Plus}

The end of the $O 3$ Run will mark the beginning of the Advanced Virgo Plus project which, in 
two separate phases, will pursue the increase of the sensitivity of the detector addressing several technical noises and improving the configuration of the detector itself.

Phase I In this first phase, which will lead to the $O 4$ Run, the purpose will be to hit the thermal noise wall, and the main upgrades will be the following: the installation of the Signal Recycling mirror, which will lead finally to have a dual-recycled interferometer (in the "broadband" configuration) with a much better sensitivity in the mid-to-high frequency region. The input laser power will be consistently increased, up to $40 \mathrm{~W}$, which will improve again the shot noise level; this will require to tackle the bigger thermal issues and increased radiation pressure noise as well. Finally, the squeezing system will become frequency-dependent, which means that it will be possible to gain in the whole frequency band without the detrimental effects which may arise at low frequency with the standard, frequency-independent squeezing.
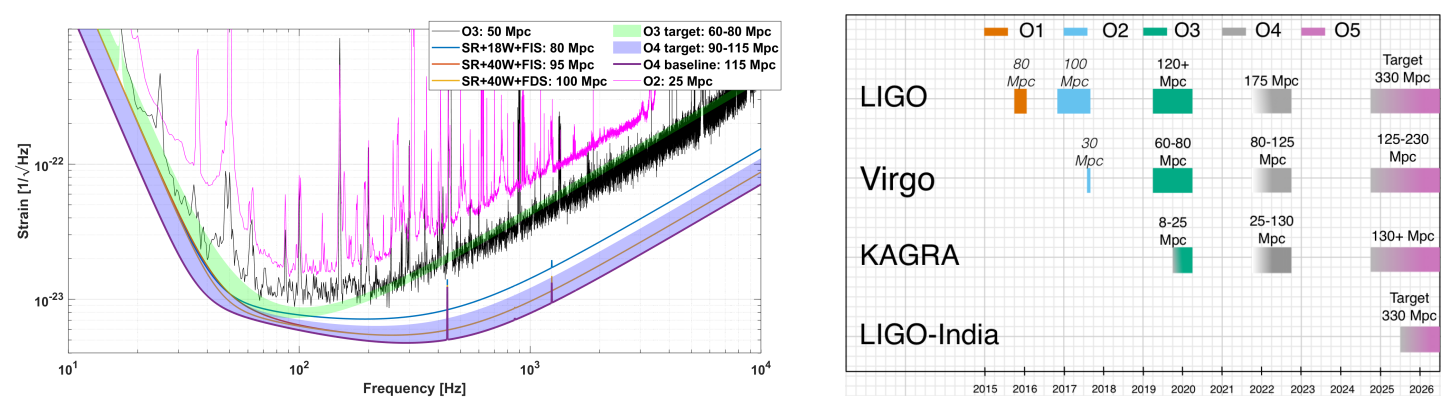

Figure 8: Projected sensitivity curve (Phase I only) and roadmap of Advanced Virgo Plus.

Phase II The second phase will happen later, and will lead to the O5 Run; here the aim of the development will be to push down the thermal noise wall itself, which will be achieved by increasing the beam size and the installation of bigger and heavier End mirrors. The mirrors will be changed also in their coatings, if the R\&D currently ongoing will produce good results. Lastly, the input laser power will be further increased, up to $100 \mathrm{~W}$.

In the meantime, the Japanese KAGRA interferometer is expected to join the international gravitational network, allowing for even more precise localization of the sources of gravitational waves, and increasing the duty cycle and the overall sensitivity of the network itself.

\section{References}

[1] B. P. Abbott et al. [LIGO Scientific and Virgo Collaborations], Phys. Rev. Lett. 119, no. 14, 141101 (2017) doi:10.1103/PhysRevLett.119.141101 [arXiv:1709.09660 [gr-qc]].

[2] B. P. Abbott et al. [LIGO Scientific and Virgo Collaborations], Phys. Rev. Lett. 119, no. 16, 161101 (2017) doi:10.1103/PhysRevLett.119.161101 [arXiv:1710.05832 [gr-qc]].

[3] B. P. Abbott et al. [LIGO Scientific and Virgo Collaborations], Phys. Rev. X 9, no. 3, 031040 (2019) doi:10.1103/PhysRevX.9.031040 [arXiv:1811.12907 [astro-ph.HE]].

[4] C. M. Caves, Phys. Rev. D 23, 1693 (1981). doi:10.1103/PhysRevD.23.1693

[5] GraceDB, https://gracedb.ligo.org 